\section{Transcriptomic Analysis Reveals the Molecular Mechanisms of Drought-Stress-Induced Decreases in Camellia sinensis Leaf Quality}

\author{
Weidong Wang, Huahong Xin, Mingle Wang, Qingping Ma, Le Wang, Najeeb A. Kaleri, \\ Yuhua Wang * and Xinghui Li *
}

College of Horticulture, Nanjing Agricultural University, Nanjing, China

The tea plant [Camellia sinensis (L.) O. Kuntze] is an important commercial crop rich in bioactive ingredients, especially catechins, caffeine, theanine and other free amino acids, which the quality of tea leaves depends on. Drought is the most important environmental stress affecting the yield and quality of this plant. In this study, the effects of drought stress on the phenotype, physiological characteristics and major bioactive ingredients accumulation of $C$. sinensis leaves were examined, and the results indicated that drought stress resulted in dehydration and wilt of the leaves, and significant decrease in the total polyphenols and free amino acids and increase in the total flavonoids. In addition, HPLC analysis showed that the catechins, caffeine, theanine and some free amino acids in C. sinensis leaves were significantly reduced in response to drought stress, implying that drought stress severely decreased the quality of $C$. sinensis leaves. Furthermore, differentially expressed genes (DEGs) related to amino acid metabolism and secondary metabolism were identified and quantified in $C$. sinensis leaves under drought stress using high-throughput Illumina RNA-Seq technology, especially the key regulatory genes of the catechins, caffeine, and theanine biosynthesis pathways. The expression levels of key regulatory genes were consistent with the results from the HPLC analysis, which indicate a potential molecular mechanism for the above results. Taken together, these data provide further insights into the mechanisms underlying the change in the quality of C. sinensis leaves under environmental stress, which involve changes in the accumulation of major bioactive ingredients, especially catechins, caffeine, theanine and other free amino acids.

Keywords: Camellia sinensis, drought stress, quality, secondary metabolite, RNA-Seq, molecular mechanisms

\section{INTRODUCTION}

Tea plant [Camellia sinensis (L.) O. Kuntze] is an important perennial, evergreen, woody crop that is grown worldwide, and its young leaves are processed to prepare a popular non-alcoholic beverage known as "tea." C. sinensis plants experienced the effects of various abiotic stresses during their lifecycle, such as drought stress (Das et al., 2012), temperature stress, salinity stress (Li et al., 2010), heavy-metal stress (Basak et al., 2001), and soil nutrient deficiency (Upadhyaya and Panda, 2013). Among these stresses, drought stress is an important factor that significantly constrains 
the yield and quality of tea products, and damage caused by drought has become increasingly frequent and unpredictable due to global climate changes, especially growing water scarcity (Zhou et al., 2014). At present, research on the effects of drought stress in C. sinensis plants is focused on the mechanisms underlying the stress response, which includes morphological, physiological, and molecular changes (Upadhyaya et al., 2013). For example, Zhou et al. (2014) and Upadhyaya et al. (2013) reported that the amounts of $\mathrm{H}_{2} \mathrm{O}_{2}$, chlorophyll, proline, and MDA and the activities of enzymes such as SOD, CAT, POX, GR, and PPO changed in $C$. sinensis plants under drought stress. In addition, differentially expressed genes related to the drought response were identified using suppression subtractive hybridization and cDNA-AFLP technology (Das et al., 2012; Gupta et al., 2013). Furthermore, two-dimensional electrophoresis identified proteins that are differentially expressed in response to drought stress (Lin et al., 2014). In contrast, there are only few studies have focused on the effects of drought stress on C. sinensis leaf quality, especially the changes in its main bioactive ingredients, such as catechins, caffeine, theanine and other free amino acids.

The production of secondary metabolites in C. sinensis plants contribute to the rich flavors, clean taste, and nutrient content of tea, and these metabolites are known to be beneficial to human health ( $\mathrm{Li}$ et al., 2015a). Among them, polyphenols (especially catechins), caffeine, and theanine are the most important constituents that determine the quality of C. sinensis leaves, which further determines the quality of tea products (Liang et al., 1990). Previous studies have shown that a poor growth environment strongly reduces the quality of $C$. sinensis leaves by changing the amounts of polyphenols, caffeine, and theanine (Ahmed et al., 2014; Zhang et al., 2014). For example, Zheng et al. (2008) reported that excessive UV$B$ irradiation suppressed the accumulation of tea catechins, and Zhang et al. (2014) demonstrated that the accumulation of individual catechins, caffeine and free amino acids was influenced by light intensity and temperature. Similarly, the amounts of bioactive ingredients in C. sinensis leaves, such as polyphenols, caffeine and free amino acids, were found to decrease in response to an extreme acid-rain environment (Duan et al., 2012). In addition, preliminary experimental results showed that drought stress affected the accumulation of polyphenols and individual bioactive ingredients, which decreased the quality of $C$. sinensis leaves (Jeyaramraja et al., 2003; Chen et al., 2010). However, direct and detailed evidence of these negative impacts is limited. On the other hand, the molecular mechanisms of major metabolic pathways in $C$. sinensis have been a focus of study, and efforts have been made to identify the key genes involved in several major metabolic pathways in C. sinensis (Li et al., 2015a). For example, Shi et al. (2011) elucidated the gene network responsible for the regulation of the secondary metabolite biosynthetic pathways in C. sinensis using high-throughput Illumina RNA-Seq technology, especially the flavonoid biosynthesis pathway (namely, the catechins biosynthesis pathway in C. sinensis), the caffeine biosynthesis pathway and the theanine biosynthesis pathway. Recently, Li et al. (2015a) analyzed the gene expression profiles related to secondary metabolic pathways in different tissues at different developmental stages in C. sinensis, which further revealed how secondary metabolic pathways are regulated during plant development and growth cycles. However, the molecular mechanisms underlying the effects of environmental stress (especially drought stress) on the accumulation of secondary metabolites in $C$. sinensis, such as catechins, caffeine, theanine and other free amino acids, remain unknown.

In the present study, we investigated the effects of drought stress on the phenotype, physiological characteristics and major bioactive ingredients accumulation in C. sinensis leaves, including the total polyphenol, flavonoid and free amino acid content. In addition, the changes in the levels of catechins, caffeine, theanine and other amino acids in C. sinensis leaves were detected by HPLC after treatment with drought stress. Furthermore, to investigate the molecular mechanisms of decrease in quality of tea leaves under drought stress, differentially expressed genes (DEGs) related to amino acid metabolism and secondary metabolism in C. sinensis plants in response to drought stress, especially genes associated with the catechin, caffeine and theanine biosynthesis pathways were identified analyzed. These data were used to explore the molecular mechanisms underlying the changes in the accumulation of the main bioactive ingredients that occur in response to drought stress and influence leaf quality in C. sinensis.

\section{MATERIALS AND METHODS}

\section{Plant Materials}

Two-year-old tea plants [Camellia sinensis (L.) O. Kuntze cv. "Longjingchangye"] were pre-incubated under normal conditions $\left(25 \pm 1{ }^{\circ} \mathrm{C}, 12\right.$-h light/12-h dark cycle) for 2 weeks in an artificial climate chamber. The drought stress assays were then carried out using 20\% (w/v) polyethylene glycol (PEG) 6000 with all of the other environmental conditions remaining constant. The first and second tender leaves from about $120^{\circ} \mathrm{C}$. sinensis plants were randomly collected at several time points $(0,2,12,24$, and $48 \mathrm{~h})$ under control or stress conditions, and the samples were immediately frozen in liquid nitrogen and stored at $-80^{\circ} \mathrm{C}$ for further analysis. Additionally, following 0, 2, and 5 days of treatment, C. sinensis leaves were randomly collected, freeze-dried and ground to a fine powder to analyze the effects of drought stress on the metabolism of the main chemical components.

\section{Determination of Chlorophyll, Malondialdehyde, and Relative Water Content}

During the period of drought stress, treated leaves were randomly sampled at 0,2 , and 5 days from about $120^{\circ} \mathrm{C}$. sinensis plants after the application of $20 \%$ PEG 6000, and the samples were used to determine the physiological characteristics. Relative water content (RWC) was measured to detect the effects of drought stress on $C$. sinensis plants according to Upadhyaya et al. 
(2008). The content of chlorophyll and malondialdehyde (MDA) in the leaves were determined by spectrophotometer method developed by Knudson et al. (1977) and Dhindsa et al. (1981), respectively.

\section{Determination of Total Polyphenol, Flavonoid, and Free Amino Acid Content}

The total polyphenols were extracted from the powdered leaf samples in $70 \%(\mathrm{v} / \mathrm{v})$ methanol at $70^{\circ} \mathrm{C}$, and the content was assessed using a UV-5200 spectrophotometer (METASH, China) at $765 \mathrm{~nm}$ according to the Folin-Ciocalteu method described by Li et al. (2015b). The total flavonoids and free amino acids were extracted in deionized water at $100^{\circ} \mathrm{C}$, and the flavonoid content was determined according to the $\mathrm{AlCl}_{3}$ method (Lin and Tang, 2007) and ninhydrin coloration method (Zheng et al., 2015).

\section{Quantification of Catechins and Caffeine by HPLC}

The catechin and caffeine content was determined by highperformance liquid chromatography (HPLC) according to Chen et al. (2015) with some modifications. Briefly, $0.2 \mathrm{~g}$ of powdered leaves was extracted with $10 \mathrm{~mL}$ of $70 \%(\mathrm{v} / \mathrm{v})$ methanol at $70^{\circ} \mathrm{C}$ for $20 \mathrm{~min}$, and the extract was then filtered through a 0.45 $\mu \mathrm{m}$ Millipore filter before being injected into an Shimadzu LC20A HPLC system (Shimadzu, Japan). A 5- $\mu$ L volume of filtrate was injected into the HPLC system and analyzed on an Inertsil ODS-SP C18 analytical column $(250 \times 4.6 \mathrm{~mm}$ i.d., $5 \mu \mathrm{m}$ nominal particle size). A solution containing $9 \%(\mathrm{v} / \mathrm{v})$ methyl cyanide, $2 \%(\mathrm{v} / \mathrm{v})$ acetic acid, and $0.02 \%(\mathrm{~m} / \mathrm{v})$ EDTA was used as mobile phase A, and mobile phase B consisted of $80 \%(\mathrm{v} / \mathrm{v})$ methyl cyanide, $2 \%(\mathrm{v} / \mathrm{v})$ acetic acid and $0.02 \%(\mathrm{~m} / \mathrm{v})$ EDTA. The samples were eluted at $35^{\circ} \mathrm{C}$ at a flow-rate of $1 \mathrm{~mL} / \mathrm{min}$, and a continuous eluent gradient was adopted to enhance peak separation. The absorbance at $278 \mathrm{~nm}$ was used to monitor peak intensities in real-time, and the peaks were identified by comparing the retention times for the sample to those of authentic standards. Authentic standards for epigallocatechin gallate (EGCG, $\geq 95 \%$ ), epicatechin gallate (ECG, $\geq 98 \%$ ), gallocatechin (GC, $\geq 98 \%$ ), epigallocatechin (EGC, $\geq 95 \%$ ), catechin (Cat, $\geq 97 \%$ ), epicatechin (EC, $\geq 98 \%$ ), gallocatechin gallate (GCG, $\geq 98 \%$ ), and caffeine ( $\geq 95 \%$ ) were purchased from Sigmae-Aldrich (St. Louis, MO, USA).

\section{Extraction and Quantitative Analysis of Theanine and Other Amino Acids}

Theanine was extracted from the samples with deionized water for $45 \mathrm{~min}$ in a water bath at $80^{\circ} \mathrm{C}$, and the extract was filtered through a $0.45-\mu \mathrm{m}$ Millipore filter before HPLC analysis. The theanine was then detected using an Shimadzu LC-20A HPLC system (Shimadzu, Japan) according to the method described by Tai et al. (2015). In addition, other amino acids were extracted as described by Wan et al. (2015) with modifications, and 17 common amino acids were identified using an L-8900 automatic amino acid analyzer (Hitachi, Japan).

\section{RNA Extraction, Library Construction, and RNA-Seq}

Total RNA from C. sinensis leaves was extracted using RNAiso Plus (TaKaRa, Japan). The integrity and quality of RNA was measured using a 2100 Bioanalyzer RNA Nano chip device (Agilent, Santa Clara, CA, USA) and a NanoDrop ND-1000 spectrophotometer (NanoDrop, Wilmington, DE). Equal amounts of RNA from three biological replicates were pooled prior to cDNA preparation. The cDNA libraries were constructed and sequenced using an Illumina HiSeq ${ }^{\mathrm{TM}} 2000$ located at the Beijing Genomics Institute (BGI, Shenzhen, China; http://www.genomics.cn/index). The data were analyzed according to the method described by Ren et al. (2014). Briefly, clean reads were obtained by removing adaptor sequences, reads in which the percentage of unknown nucleotides $(\mathrm{N})$ was greater than $5 \%$ and low quality reads (The rate of reads which quality value $\leq 10$ is more than $20 \%$ ). The clean reads were then assembled into Unigenes using the Trinity software (Grabherr et al., 2011). Finally, blastx alignment $(e<0.00001)$ between Unigenes and protein databases like NR, Swiss-Prot, KEGG and COG were performed, and the best aligning results are used to decide sequence direction of Unigenes.

\section{Identification of DEGs Related to Amino Acid Metabolism and Secondary Metabolism}

Unigene expression was calculated using the FPKM method, and the differentially expressed genes (DEGs) were identified according to stringent criteria: a $P<0.05$, an FDR $\leq 0.001$ and a $\mid \log _{2}$ Ratio $\mid \geq 1.0$. The DEGs were then subjected to KEGG Ontology (KO) enrichment analysis based on a hypergeometric test. In addition, DEGs related to the metabolism of major amino acids (e.g., theanine, glutamate, and alanine) and secondary metabolites (e.g., catechins, flavone, and caffeine metabolism) were identified from KEGG annotation, and a hierarchical clustering analysis was then carried out using Cluster 3.0 software. To investigate the molecular mechanisms of drought stress affecting the accumulation of main bioactive ingredients, DEGs related to three major metabolic pathways in C. sinensis, flavonoids, caffeine and theanine biosynthesis pathway, were selected for more detailed analyses.

\section{Quantitative Real-Time PCR (qRT-PCR) Analysis of the Selected DEGs}

qRT-PCR was used to confirm the accuracy of the differential expression of DEGs via RNA-Seq. Total RNA was isolated from the leaf samples and treated with DNase I to remove any genomic DNA contamination. The single-stranded cDNAs used for realtime PCR analysis were synthesized using a PrimeScript ${ }^{T M}$ RT Reagent Kit with gDNA Eraser (TaKaRa, Dalian, China). qRT-PCR was carried out using SYBR Premix Ex TaqTM II (TaKaRa, Dalian, China) on an Eppendorf Real-Time PCR System (Mastercycler ${ }^{\circledR}$ ep realplex, Germany) according to the manufacturer's protocol, and the amplification was conducted as described by Ren et al. (2014). The C. sinensis $\beta$-actin gene (Cs $\beta$-actin, GenBank: HQ420251.1) was amplified as an internal 
A

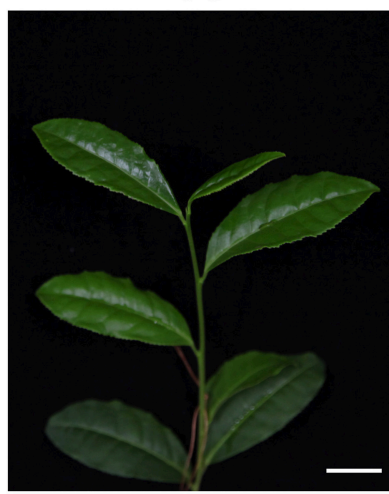

B

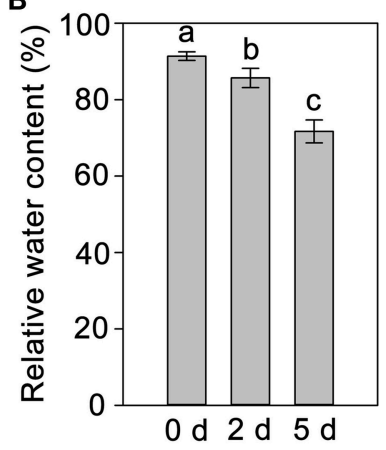

$2 d$

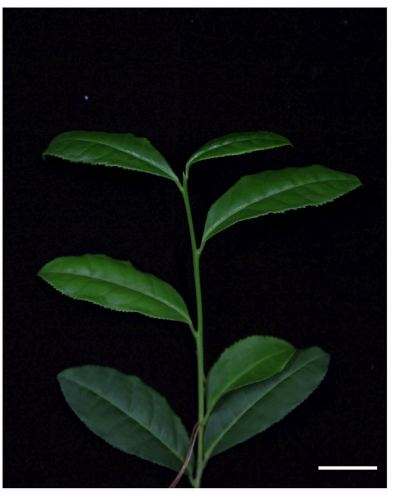

C

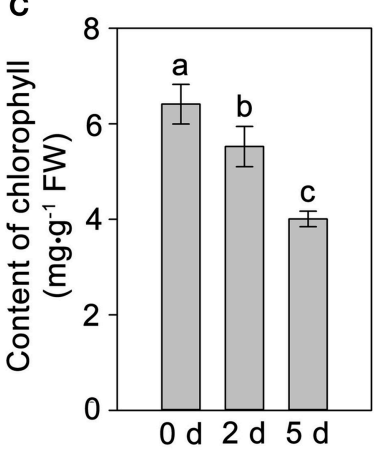

$5 d$

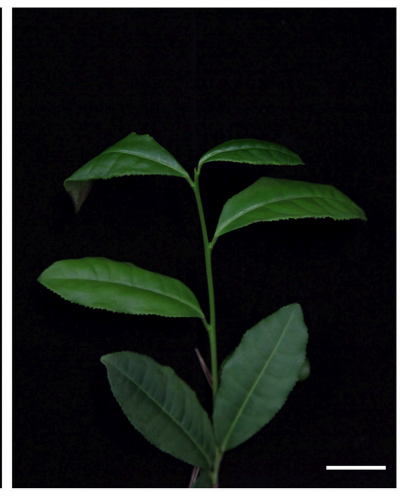

D

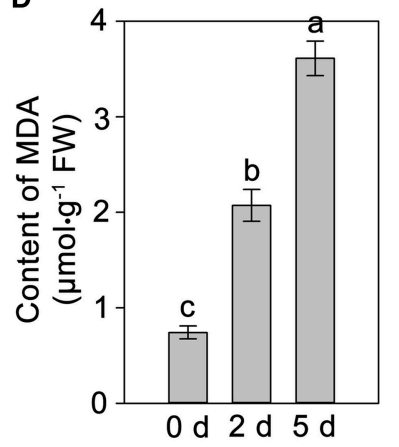

FIGURE 1 | Effects of drought stress on the phenotype and physiological characteristics of $\boldsymbol{C}$. sinensis leaves. The leaves begun to wilt at 2 days under drought stress, the wilting degree of leaves deepened and exhibited scorch in leaf tips at 5 days (A). The RWC and chlorophyll content in $C$. sinensis leaves decreased rapidly $(\mathbf{B}, \mathbf{C})$ and the content of MDA increased significantly (D) at 2 and 5 days after treated with drought stress.

reference standard, and the relative expression levels were calculated using the $2^{-\Delta \Delta C T}$ method (Livak and Schmittgen, 2001). Three biological and three technical replicates were performed for each sample, and the primers used for qRT-PCR are listed in Supplementary S1.

\section{Statistical Analyses}

Each experiment was repeated at least three times, and all data are expressed as the means \pm standard deviations (SD). Group differences were tested using one-way ANOVA and Duncan's test, and significant differences among various treatment groups are represented by different letters $(P<0.05)$. The data were analyzed using SPSS 20 software.

\section{RESULTS}

\section{Changes in Total Polyphenol, Flavonoid, and Free Amino Acid Content}

The phenotypic changes of $C$. sinensis leaves in response to drought stress were recorded at 0,2 , and 5 days. The leaves of $C$. sinensis plants had begun to wilt after 2 days of drought stress, and, the wilting degree of leaves deepened and the leaf tips exhibited scorch at 5 days (Figure 1A). In addition, the analysis of physiological characteristics showed that the RWC and chlorophyll content in C. sinensis leaves decreased rapidly
(Figures 1B,C) and the content of MDA increased significantly (Figure 1D) at 2 and 5 days after treated with drought stress, implying drought stress obviously affected the normal growth of C. sinensis plants, particularly its leaves. Simultaneously, the total polyphenols, flavonoids and free amino acids in the leaf samples were measured after drought stress at different time points $(0,2$, and 5 days). As shown in Figure 2, the total polyphenol content significantly and rapidly decreased after drought stress at 2 and 5 days compared with the control plants (0 days). The total free amino acid content showed similar decreases in response to drought stress. Interestingly, drought stress significantly increased the content of total flavonoids.

\section{The Effect of Drought Stress on Catechins and Caffeine Content}

A typical HPLC profile of detected individual catechins and caffeine shows seven individual catechins and caffeine (Supplementary S2), and the changes in the individual catechin components and caffeine in leaf samples after different durations of drought stress are shown in Supplementary S3. The concentrations of individual catechins and caffeine among different time points were compared, as shown in Table 1. Specifically, the amounts of gallocatechin (GC), epigallocatechin (EGC), catechin (Cat), and epicatechin (EC) significantly 
TABLE 1 | Drought stress-induced changes in the amount of individual catechins and caffeine $\left(\mathrm{mg} \cdot \mathrm{g}^{-1} \mathrm{DW}\right)$.

\begin{tabular}{|c|c|c|c|c|c|c|c|c|}
\hline Treatment time (d) & GC & EGC & Cat & EC & EGCG & ECG & GCG & Caffeine \\
\hline 0 & $3.47 \pm 0.03 a$ & $24.8 \pm 0.29 a$ & $14.18 \pm 1.07 a$ & $9.74 \pm 0.19 a$ & $59.84 \pm 1.82 a$ & $14.78 \pm 0.85 a$ & $5.40 \pm 0.05 a$ & $26.36 \pm 0.03 a$ \\
\hline 2 & $3.20 \pm 0.02 b$ & $23.24 \pm 0.82 b$ & $9.52 \pm 0.08 b$ & $8.43 \pm 0.12 b$ & $18.94 \pm 0.05 c$ & $4.07 \pm 0.04 c$ & $3.81 \pm 0.10 c$ & $23.15 \pm 0.18 b$ \\
\hline 5 & $3.02 \pm 0.02 c$ & $19.46 \pm 0.10 c$ & $10.21 \pm 0.11 b$ & $8.84 \pm 0.08 b$ & $36.50 \pm 0.44 b$ & $10.69 \pm 0.11 b$ & $4.34 \pm 0.06 b$ & $17.85 \pm 0.25 c$ \\
\hline
\end{tabular}

Values are presented as the mean $\pm S D$ from three independent experiments, and different letters indicate significant differences at $P<0.05$ according to Duncan's test.

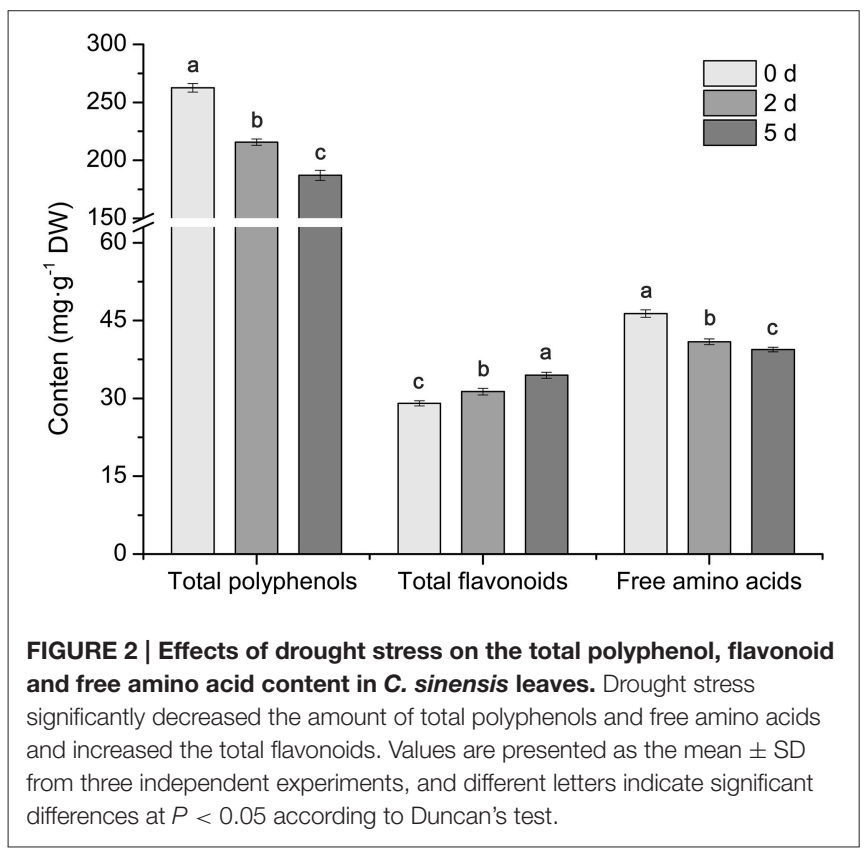

and gradually decreased after drought stress compared with the control (0 days). Unexpectedly, the concentrations of epigallocatechin gallate (EGCG), gallocatechin gallate (GCG), and epicatechin gallate (ECG) significantly decreased after 2 days of drought stress treatment and then increased slightly at 5 days; however, the overall trends were of decline. In addition, drought stress rapidly reduced the caffeine accumulation in C. sinensis leaves.

\section{The Effect of Drought Stress on Theanine and Other Amino Acid Content}

A typical HPLC profile of detected theanine is shown in Supplementary S4, and the changes in the concentration of theanine among different time points were compared, as shown in Supplementary S5. Theanine accumulation significantly and gradually decreased from $30.168 \mathrm{mg} \cdot \mathrm{g}^{-1}$ (0 days) to $28.017 \mathrm{mg} \cdot \mathrm{g}^{-1}$ (2 days) and $23.989 \mathrm{mg} \cdot \mathrm{g}^{-1}$ (5 days; Table 2). Simultaneously, the content of 17 common amino acids was measured. The levels of several amino acids changed to varying degrees after treatment with drought stress (Table 2). Specifically, the content of Glu, Gly, Met, Leu, Phe, Arg, and Lys significantly decreased after drought stress compared with the control (0 days). In contrast, drought stress treatment significantly increased Asp, Ser and Pro accumulation, especially
TABLE 2 | Drought stress-induced changes in content of theanine and other free amino acids $\left(\mathrm{mg} \cdot \mathrm{g}^{-1} \mathrm{DW}\right)$.

\begin{tabular}{lccc}
\hline Amino acid & \multicolumn{3}{c}{ Treatment time (d) } \\
\cline { 2 - 4 } & $\mathbf{0}$ & $\mathbf{2}$ & $\mathbf{5}$ \\
\hline The & $30.168 \pm 0.471 \mathrm{a}$ & $28.017 \pm 0.411 \mathrm{~b}$ & $23.989 \pm 0.411 \mathrm{c}$ \\
Asp & $1.238 \pm 0.007 \mathrm{~b}$ & $1.224 \pm 0.009 \mathrm{~b}$ & $1.259 \pm 0.006 \mathrm{a}$ \\
Thr & $0.627 \pm 0.003 \mathrm{a}$ & $0.618 \pm 0.005 \mathrm{~b}$ & $0.626 \pm 0.002 \mathrm{a}$ \\
Ser & $0.684 \pm 0.003 \mathrm{~b}$ & $0.690 \pm 0.009 \mathrm{ab}$ & $0.698 \pm 0.003 \mathrm{a}$ \\
Glu & $1.711 \pm 0.009 \mathrm{a}$ & $1.638 \pm 0.019 \mathrm{~b}$ & $1.564 \pm 0.010 \mathrm{c}$ \\
Gly & $0.771 \pm 0.007 \mathrm{a}$ & $0.735 \pm 0.006 \mathrm{~b}$ & $0.717 \pm 0.005 \mathrm{c}$ \\
Ala & $0.776 \pm 0.006 \mathrm{a}$ & $0.757 \pm 0.006 \mathrm{~b}$ & $0.783 \pm 0.005 \mathrm{a}$ \\
Cys & $0.136 \pm 0.004 \mathrm{a}$ & $0.134 \pm 0.002 \mathrm{a}$ & $0.139 \pm 0.003 \mathrm{a}$ \\
Val & $0.763 \pm 0.004 \mathrm{ab}$ & $0.755 \pm 0.004 \mathrm{~b}$ & $0.765 \pm 0.006 \mathrm{a}$ \\
Met & $0.162 \pm 0.001 \mathrm{a}$ & $0.153 \pm 0.004 \mathrm{~b}$ & $0.153 \pm 0.003 \mathrm{~b}$ \\
Ile & $0.597 \pm 0.003 \mathrm{ab}$ & $0.591 \pm 0.003 \mathrm{~b}$ & $0.602 \pm 0.004 \mathrm{a}$ \\
Leu & $1.203 \pm 0.004 \mathrm{a}$ & $1.176 \pm 0.006 \mathrm{c}$ & $1.188 \pm 0.006 \mathrm{~b}$ \\
Tyr & $0.397 \pm 0.007 \mathrm{a}$ & $0.391 \pm 0.007 \mathrm{a}$ & $0.395 \pm 0.006 \mathrm{a}$ \\
Phe & $0.777 \pm 0.001 \mathrm{a}$ & $0.758 \pm 0.008 \mathrm{~b}$ & $0.743 \pm 0.005 \mathrm{c}$ \\
Lys & $0.996 \pm 0.007 \mathrm{a}$ & $0.991 \pm 0.006 \mathrm{ab}$ & $0.981 \pm 0.005 \mathrm{~b}$ \\
His & $0.322 \pm 0.001 \mathrm{a}$ & $0.322 \pm 0.002 \mathrm{a}$ & $0.323 \pm 0.003 \mathrm{a}$ \\
Arg & $0.705 \pm 0.006 \mathrm{a}$ & $0.691 \pm 0.006 \mathrm{~b}$ & $0.693 \pm 0.005 \mathrm{~b}$ \\
Pro & $0.649 \pm 0.003 \mathrm{c}$ & $0.740 \pm 0.005 \mathrm{~b}$ & $0.853 \pm 0.009 \mathrm{~b}$ \\
& &
\end{tabular}

Values are presented as the mean $\pm S D$ from three independent experiments, and different letters indicate significant differences at $P<0.05$ according to Duncan's test.

Pro accumulation; this accumulation may be closely related to the drought tolerance of C. sinensis. Cys, Val, Ile, Tyr, His, Thr, and Ala did not show marked level changes in response to drought stress.

\section{DEGs Related to Amino Acid Metabolism and Secondary Metabolism}

Based on the KEGG database, 572 and 661 DEGs were annotated and found to be associated with amino acid metabolism and secondary metabolism pathways, respectively (Supplementary S6, S7). As shown in Figure 3A, the total number of DEGs related to various amino acid metabolic pathways increased strongly and reached 420 at $24 \mathrm{~h}$ under drought stress; thereafter, it decreased to 384 at $48 \mathrm{~h}$. Interestingly, the number of DEGs related to the phenylalanine metabolic pathway, a key regulatory pathway in the metabolism of the main chemical components in C. sinensis leaves, exhibited most obviously (Figure 3A). Similar DEGs change pattern were also observed for the total number of DEGs associated with the metabolic 


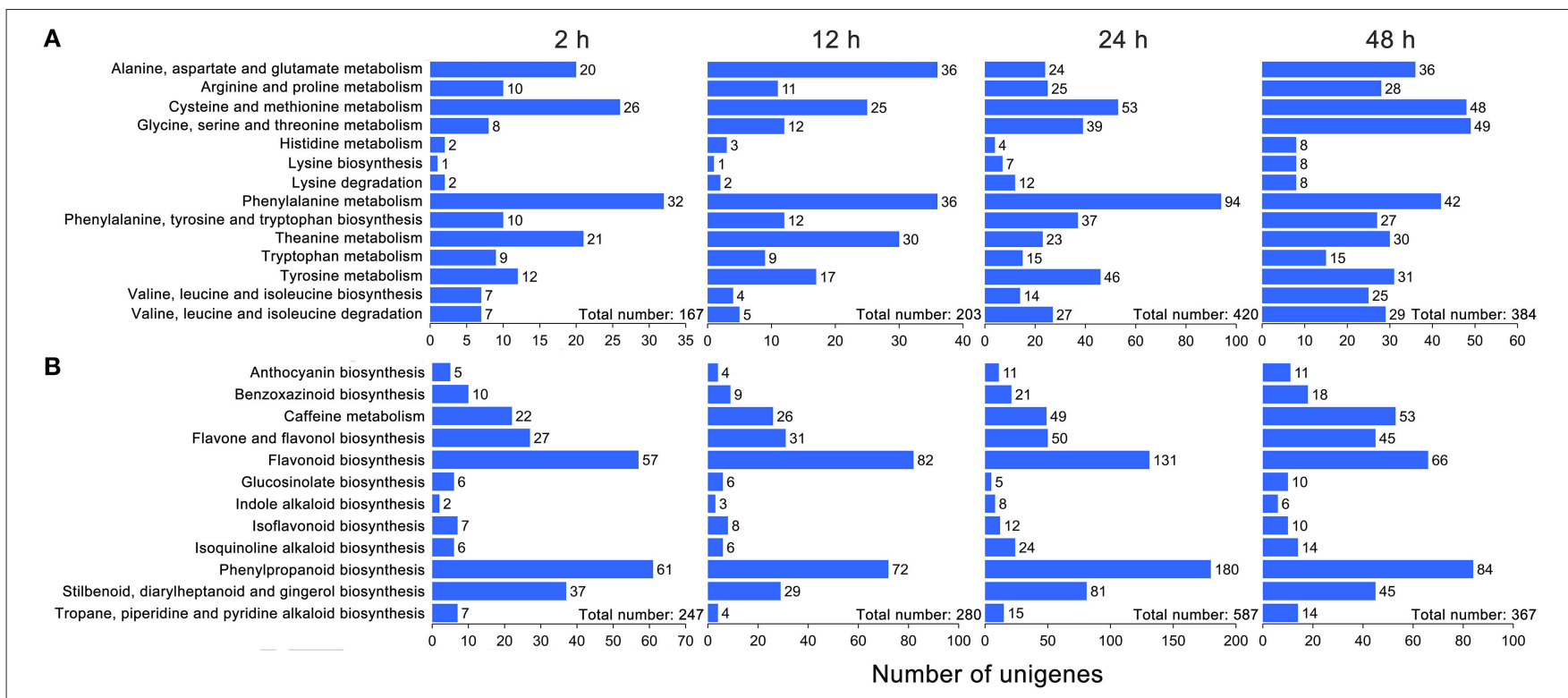

FIGURE 3 | KEGG ontology (KO) enrichment analysis of DEGs related to amino acid metabolism and secondary metabolism in C. sinensis leaves under drought stress. Five hundred and seventy-two and 661 DEGs were annotated and found to be associated with amino acid metabolism (A) and secondary metabolism (B), respectively, based on the KEGG database.

pathways of various secondary metabolites, particularly the key regulatory genes of flavonoid and phenylpropanoid biosynthesis pathways, which affect tea quality by regulating the metabolism of polyphenols and aromatic substances (Figure 3B). In addition, the hierarchical clustering analysis showed that strong changes in DEG expression levels were observed at $24 \mathrm{~h}$ of drought stress (Figure 4), implying that $24 \mathrm{~h}$ may be an important time point in the regulation of genes related to amino acid metabolism and secondary metabolism; this interpretation is consistent with the changes observed over time in the total number of DEGs.

\section{Changes in the Flavonoid Biosynthesis Pathway in C. sinensisin Response to Drought Stress}

Polyphenols are the most important class of secondary metabolites in C. sinensis that includes flavan-3-ols (catechins), flavones, flavonols, isoflavones, flavanones, dihydroflavonols, and anthocyanidins synthesized by the flavonoid biosynthesis pathway, which is one of the most characterized secondary metabolic routes in plant systems (Yang et al., 2015). Here, the transcriptome analysis identified 103 DEGs involved in the flavonoid biosynthesis pathway, and the details of these DEGs are listed in Supplementary S8. As shown in Figure 5A, almost all of the known genes related to the flavonoid biosynthesis pathway were detected under drought stress, such as phenylalanine ammonia lyase (PAL, 23 unigenes), cinnamate 4-hydroxylase (C4H, 5 unigenes), 4-coumarate CoA ligase (4CL, 8 unigenes), chalcone synthase (CHS, 7 unigenes), chalcone isomerase (CHI, 2 unigenes), flavonoid $3^{\prime}$-hydroxylase ( $\left(3^{\prime} \mathrm{H}, 2\right.$ unigenes), flavonoid $3^{\prime}, 5^{\prime}$-hydroxylase $\left(\mathrm{F}^{\prime} 5^{\prime} \mathrm{H}, 10\right.$ unigenes), flavanone

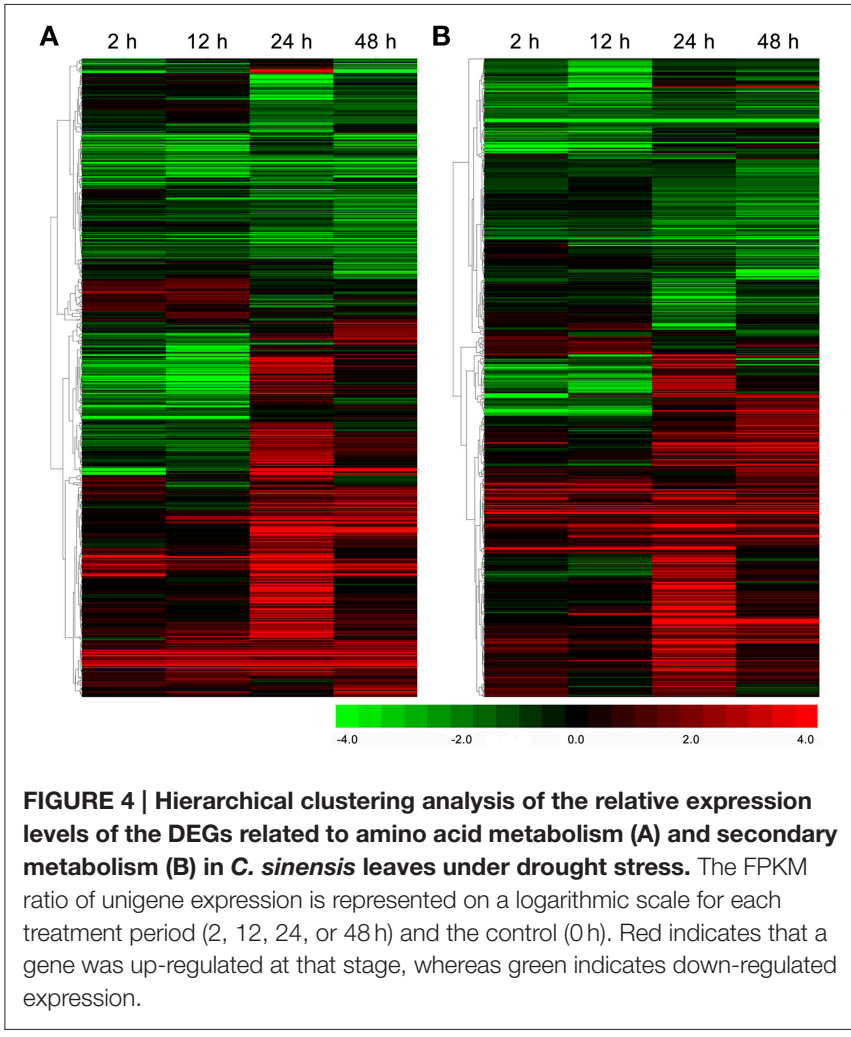

3-hydroxylase (F3H, 8 unigenes), flavonol synthase (FLS, 17 unigenes), flavone synthase (FNS, 1 unigene), dihydroflavonol 4-reductase (DFR, 10 unigenes), leucoanthocyanidin reductase (LAR, 6 unigenes), anthocyanidin synthase (ANS, 1 unigene), 
and anthocyanidin reductase (ANR, 15 unigene). In addition, the hierarchical clustering analysis showed that the FLS and FNS unigenes were continuously up-regulated under drought stress (Figure 5B), which may have been responsible for the increases in the total flavonoid content in response to drought stress (Figure 2). Interestingly, the expression levels of most unigenes, such as CHS, DFR, LAR, ANS, and ANR, tended to first decrease and then increase in response to drought stress; this pattern is consistent with the results of the HPLC analyses of the ECG, EGCG, and GCG content (Figure 5B and Table 1).

\section{Changes in the Caffeine Biosynthesis Pathway in C. sinensis under Drought Stress}

The transcriptome analysis identified 85 DEGs related to caffeine, including IMP dehydrogenase (IMPDH, 10 unigenes), Sadenosylmethionine synthase (SAMS, 6 unigenes), theobromine synthase (MXMT, 2 unigenes), and tea caffeine synthase (TCS, 69 unigenes; Figure 6A); the unigene IDs of these genes are listed in Supplementary S9. Simultaneously, the hierarchical clustering analysis showed that most members of the TCS gene family, such as the TCS1, TCS3, TCS4, TCS5, and TCS6 unigenes, were down-regulated in response to drought stress, but a few family members, predominantly TCS2 unigenes, were upregulated (Figure 6B). In addition, AMP deaminase (AMPD), $\mathrm{N}$-methylnucleotidase (N-MeNase), and 7-methylxanthosine synthase (7-NMT) were not among the DEGs associated with drought stress (Figure 6A).

\section{Changes in the Theanine Biosynthesis Pathway in C. sinensis under Drought Stress}

Theanine is a unique, non-protein-derived amino acid in the $C$. sinensis plant and is important in the production of the distinctive aroma and umami flavor of tea. As shown in Figure $7 \mathbf{A}$ and Supplementary S10, 70 genes related to theanine biosynthesis and degradation were differentially expressed under drought stress, including glutamine synthetase (GS, 33 unigenes), glutamate synthase (GOGAT, 24 unigenes), glutamate dehydrogenase (GDH, 2 unigenes), alanine aminotransferase (ALT, 1 unigene), arginine decarboxylase (ADC, 6 unigenes), theanine synthetase (TS, 4 unigenes), and theanine hydrolase (ThYD, 1 unigenes). Except for ThYD and one GS unigene, all of the unigenes were down-regulated in response to drought stress (Figure 7B), which is consistent with the observed decrease in theanine accumulation in response to drought stress.

\section{Quantitative Real-Time PCR (qRT-PCR) Validation of DEGs From RNA-Seq}

To experimentally validate the expression profiles of unigenes obtained from the Illumina RNA-Seq analysis, 15 DEGs related to flavonoid (7 DEGs), caffeine (3 DEGs), and theanine (5 DEGs) biosynthesis were selected for qRT-PCR, including FLS (Unigene61984_All), FNS (Unigene62380_All), DFR (CL632.Contig2_All and Unigene68610_All), LAR
(Unigene67547_All), ANS (Unigene93114_All), ANR (Unigene101679_All), IMPDH (Unigene63589_All), SAMS (Unigene32368_All), TCS (Unigene22943_All), GOGAT (Unigene105022_All and Unigene5788_All), ADC (Unigene38338_All), TS (Unigene10948_All), and ThYD (Unigene70558_All). The qRT-PCR outcomes in each case closely corresponded to the transcript levels estimated from the RNA-Seq output (Figure 8A). In addition, correlation analysis also showed that the expression tendency of these genes from qRT-PCR showed significant similarity $\left(R^{2}=0.93\right)$ with the Illumina RNA-Seq data (Figure 8B), suggesting the reproducibility and accuracy of the RNA-Seq results.

\section{DISCUSSION}

Drought stress significantly limits the yield and quality of tea products in tea-growing countries (Cheruiyot et al., 2010). Therefore, understanding the mechanisms underlying the effects of drought stress on C. sinensis is important. Although an increasing number of studies have explored the morphological, physiological and molecular changes that occur in C. sinensis plants in response to drought stress (Upadhyaya et al., 2008; Das et al., 2012; Zhou et al., 2014), but few have investigated the effects of drought stress on the accumulation of the main bioactive components in C. sinensis leaves (Jeyaramraja et al., 2003; Chen et al., 2010). Our investigation shows that dehydration and wilting of leaves are resulted from drought stress and the normal growth of $C$. sinensis plants was seriously affected by drought stress. Meanwhile, we found that drought stress decreased the total polyphenol and free amino acid content in C. sinensis leaves, which is consistent with the findings of Cheruiyot et al. (2007) and Chen et al. (2010), who reported that drought stress reduced C. sinensis leaf quality as indicated by a significant decrease in total polyphenol and free amino acid levels. In addition, the total flavonoids content significantly increased under drought stress in the present study, which may be an important reason that drought stress affected the liquor color of tea (Liang et al., 1990). Furthermore, HPLC analysis showed that drought stress significantly reduced the content of catechins, caffeine, theanine and some free amino acids in C. sinensis leaves. Together, these results indicate that drought stress reduces the quality of $C$. sinensis leaves by affecting the normal growth of $C$. sinensis plants and changing the accumulation of major bioactive ingredients, such as polyphenols (especially catechins), flavonoids, caffeine, theanine and other free amino acids.

Catechins account for approximately $70 \%$ of all polyphenols, which are the primary astringent substances of tea (Liu et al., 2015), in C. sinensis. In addition, numerous studies have indicated that catechins exert multiple effects on human health and play important antibacterial, antiviral, anti-radiation, and anti-aging roles (Higdon and Frei, 2003; Ho et al., 2008). Previous studies showed that catechins are abundant in the young leaves and buds of $C$. sinensis plants and include esterified catechins [such as epicatechin gallate (ECG), epigallocatechin gallate (EGCG) and gallocatechin gallate (GCG)] and non-esterified catechins [such as catechin (Cat), epicatechin (EC), gallocatechin 
A

Flavonoid biosynthesis pathway

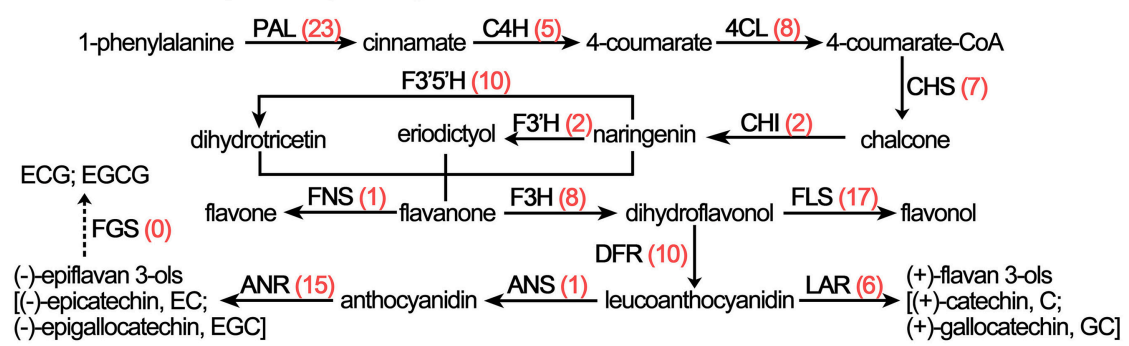

B

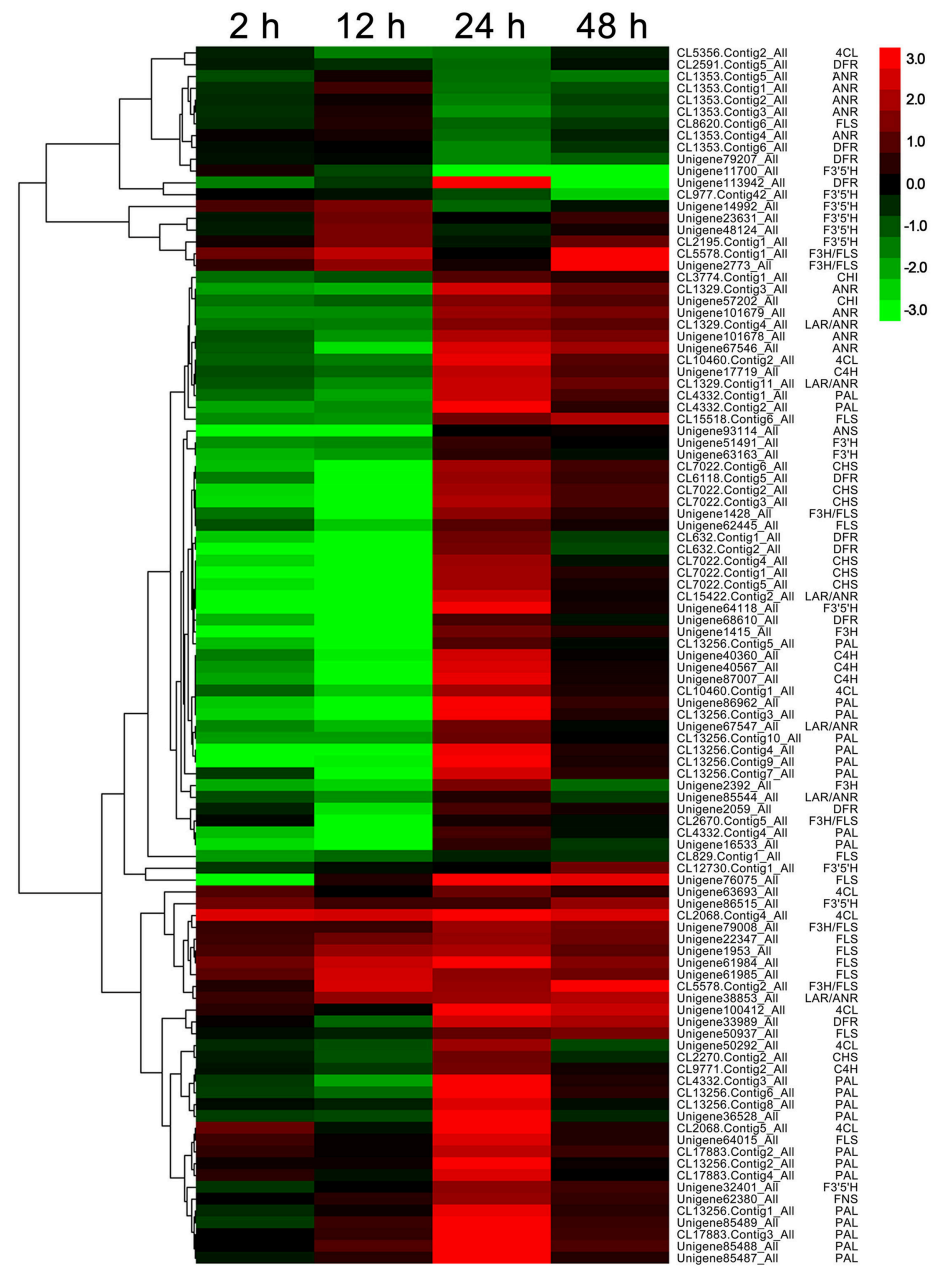

FIGURE 5 | DEGs involved in flavonoid biosynthesis in C. sinensis leaves under drought stress. (A) The flavonoid biosynthesis pathway. The red numbers in parentheses following each gene name indicate the number of corresponding DEGs. PAL, phenylalanine ammonia lyase; C4H, cinnamate 4-hydroxylase; 4CL,

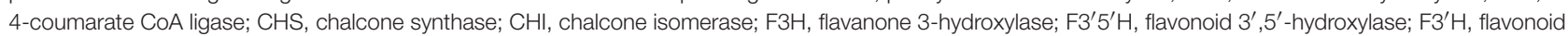
$3^{\prime}$-hydroxylase; FLS, flavonol synthase; FNS, flavone synthase; DFR, dihydroflavonol 4-reductase; ANS, anthocyanidin synthase; ANR, anthocyanidin reductase; LAR, leucoanthocyanidin reductase; FGS, flavan-3-ol gallate synthase. (B) Hierarchical clustering analysis of the relative expression levels of the DEGs related to flavonoid biosynthesis. The FPKM ratio of unigene expression is represented on a logarithmic scale for each treatment period $(2,12,24$, or $48 \mathrm{~h})$ and the control $(0 \mathrm{~h})$. Red indicates that a gene was up-regulated at that stage, whereas green indicates down-regulated expression.

(GC), and epigallocatechin (EGC); (Graham, 1992)]. However, the accumulation of catechins in C. sinensis is very susceptible to various environmental stresses, such as high ultraviolet radiation (Agati and Tattini, 2010), low temperature (Lillo et al., 2008), and drought stress (Jeyaramraja et al., 2003; Wang et al., 2012). We also found that the individual catechin content significantly decreased in response to drought stress, which is similar to the observations of Bhattacharya et al. (2015). Unexpectedly, the 


\section{A Caffeine biosynthesis pathway}

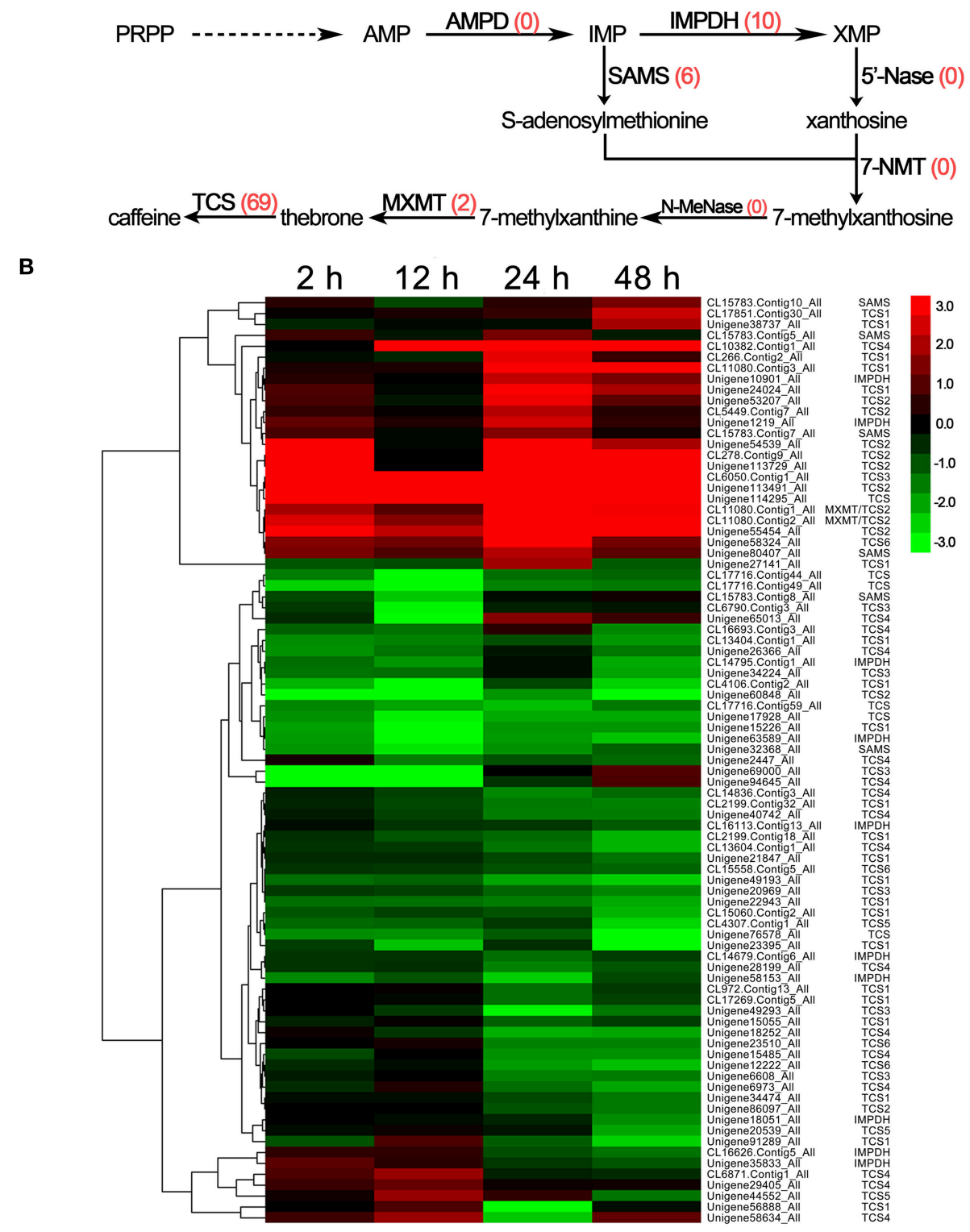

FIGURE 6 | DEGs involved in caffeine biosynthesis in C. sinensis leaves under drought stress. (A) The caffeine biosynthesis pathway. The red numbers in parentheses following each gene name indicate the number of corresponding DEGs. AMPD, AMP deaminase; IMPDH, IMP dehydrogenase; $5^{\prime}$-Nase, $5^{\prime}$-nucleotidase; SAMS, S-adenosylmethionine synthase; 7-NMT, 7-methylxanthosine synthase; N-MeNase, N-methylnucleotidase; MXMT, theobromine synthase; TCS, tea caffeine synthase. (B) Hierarchical clustering analysis of the relative expression levels of the DEGs related to caffeine biosynthetic. The FPKM ratio of unigene expression is represented on a logarithmic scale for each treatment period $(2,12,24$, or $48 \mathrm{~h})$ and the control ( $0 \mathrm{~h})$. Red indicates that a gene was up-regulated at that stage, whereas green indicates down-regulated expression.

EGCG, GCG and ECG concentrations in C. sinensis leaves tended to first decrease and then increase in response to drought stress.

Recently, key genes involved in the regulation of the flavonoid biosynthesis pathway in C. sinensis have been further recognized and characterized using RNA-Seq technology, particularly the key regulatory genes of monomeric catechins biosynthesis (Shi et al., 2011; Li et al., 2015a). However, few reports are available on the changes of genes related to catechin biosynthesis in response to environmental stresses in C. sinensis (Xiong et al., 2013). Our transcriptome analysis data indicate that almost all known genes related to flavonoid biosynthesis are differentially expressed under drought stress. Particularly, the levels of CHS, DFR, LAR, ANS, and ANR tended to decrease and subsequently increase in response to drought stress, which is consistent with the changes of ECG, EGCG, and GCG levels. These findings suggest that the effects of drought stress on the catechins contents of $C$. 


\section{A}

Theanine biosynthesis pathway

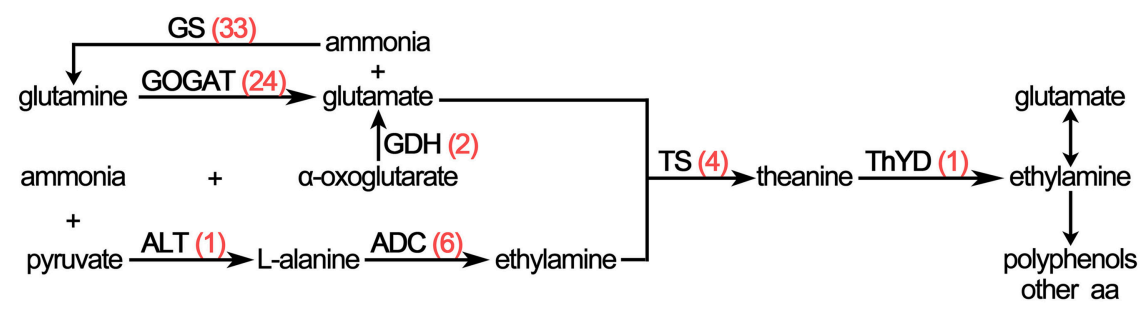

B

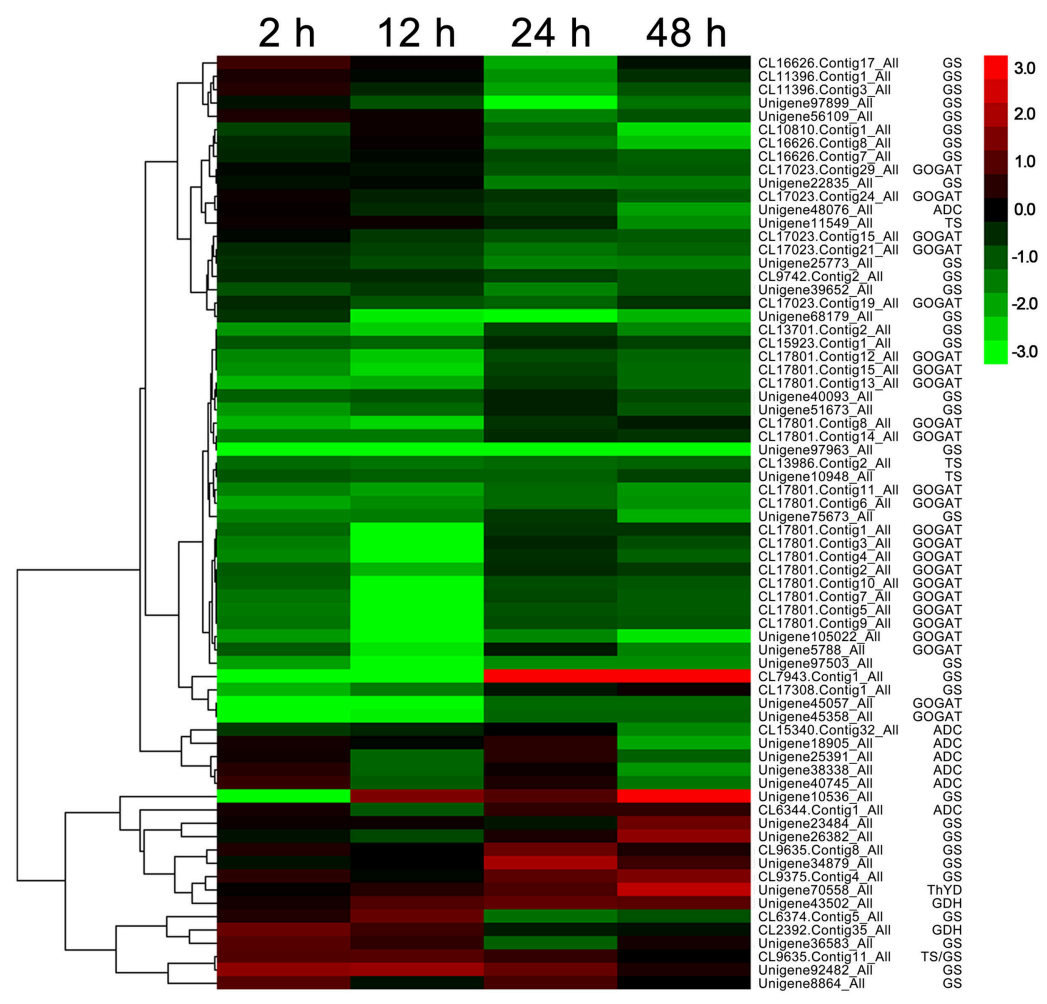

FIGURE 7 | DEGs involved in theanine biosynthesis in C. sinensis leaves under drought stress. (A) The theanine biosynthesis pathway. The red numbers in parentheses following each gene name indicate the number of corresponding DEGs. GS, glutamine synthetase; GOGAT, glutamate synthase; GDH, glutamate dehydrogenase; ALT, alanine aminotransferase; ADC, arginine decarboxylase; TS, theanine synthetase; ThYD, theanine hydrolase. (B) Hierarchical clustering analysis of the relative expression levels of the DEGs related to theanine biosynthetic. The FPKM ratio of unigene expression is represented on a logarithmic scale for each treatment period $(2,12,24$, or $48 \mathrm{~h})$ and the control $(0 \mathrm{~h})$. Red indicates that a gene was up-regulated at that stage, whereas green indicates down-regulated expression.

sinensis leaves depend on the regulation of genes that participate in flavonoid biosynthesis pathway. In addition, the FLS and FNS unigenes, which participate in flavonoid biosynthesis, were upregulated in response to drought stress. This finding explains the increases in the total flavonoid content of $C$. sinensis leaves in response to drought stress.

Caffeine (1, 3, 7-trimethylxanthine), another important bioactive ingredient in $C$. sinensis plants, is a purine alkaloid that has been widely used as stimulant or ingredient in drugs ( $\mathrm{Li}$ et al., 2015a). Caffeine is mainly synthesized in young leaves in C. sinensis via a typical caffeine biosynthetic pathway including purine biosynthesis and purine modification steps (Ashihara and
Kubota, 1986). In the present study, IMPDH, SAMS, MXMT, and TCS genes were identified, and most of these genes were downregulated in response to drought stress. This finding is consistent with the decreases in the caffeine content in $C$. sinensis leaves following drought stress. These results reveal that drought stress inhibits the expression of genes related to caffeine biosynthesis, such as IMPDH, SAMS, MXMT, and TCS, thereby reducing the accumulation of caffeine in $C$. sinensis leaves.

Theanine is generally considered to play crucial roles in producing the distinctive aroma and umami flavor of tea. Therefore, theanine content is important in determining the quality of a tea product ( $\mathrm{Mu}$ et al., 2015). Previous studies 


\section{A}
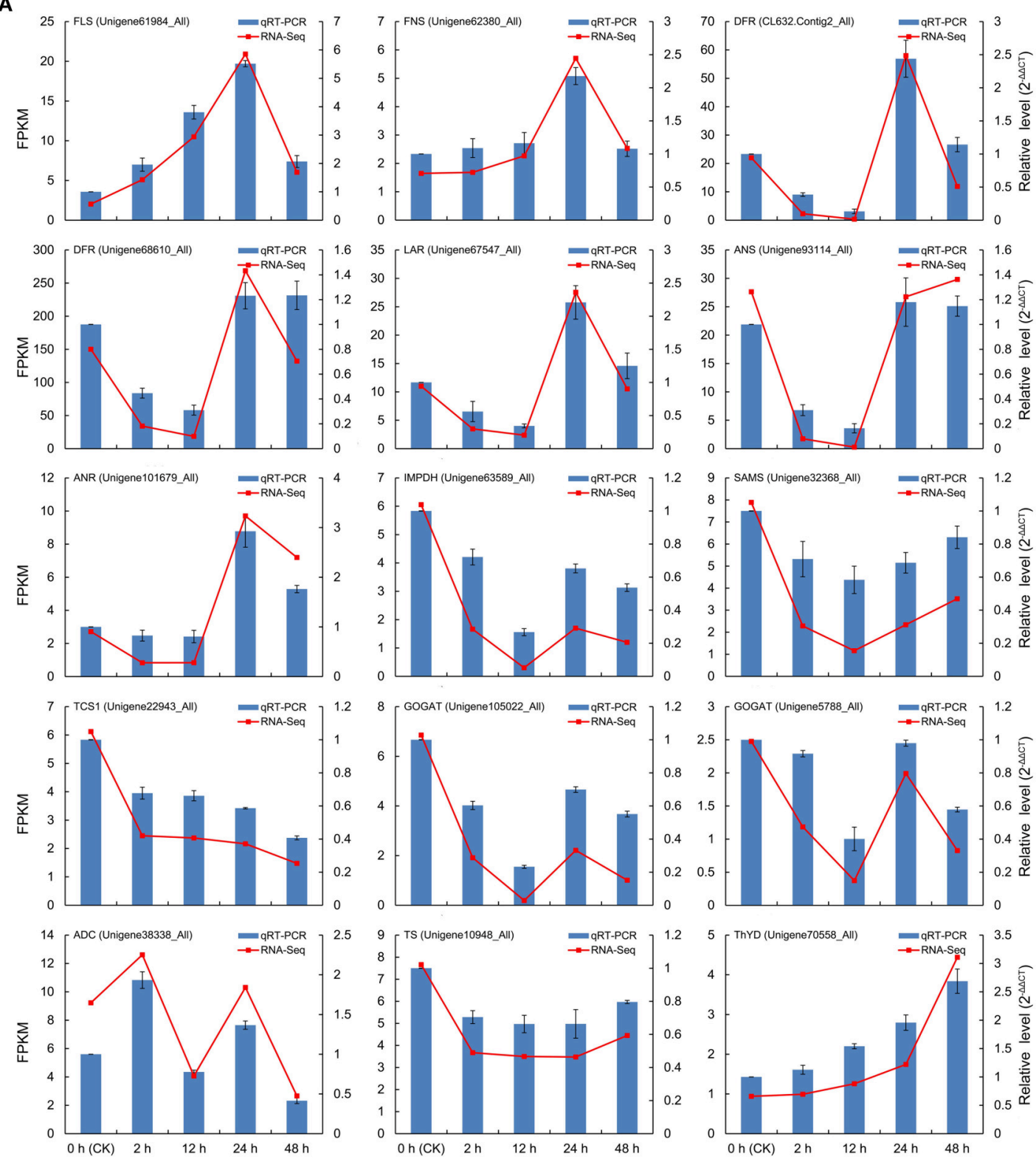

B

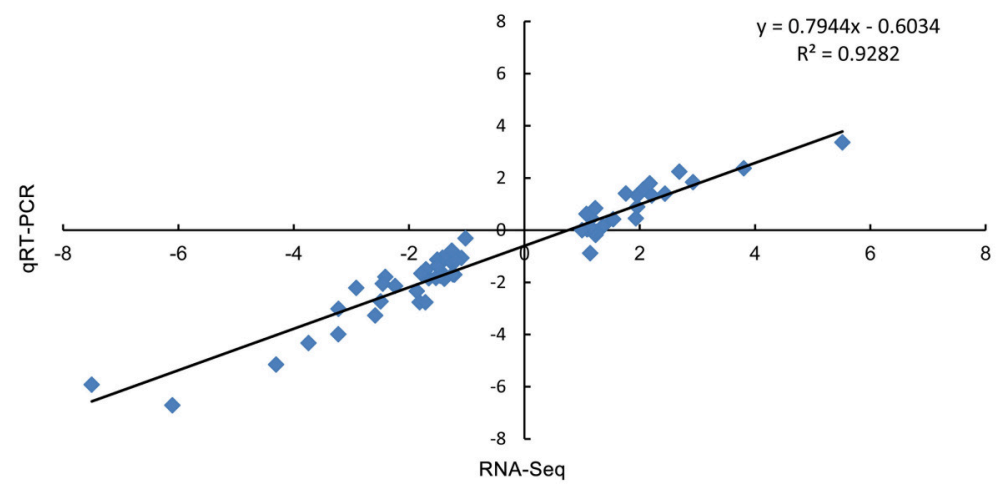

FIGURE 8 | Verification of the relative expression levels of DEGs by qRT-PCR. (A) Expression patterns of 15 DEGs related to the flavonoid, caffeine, and theanine biosynthetic pathways by qRT-PCR (blue bar) and RNA-Seq (red line). (B) Correlation of the expression levels of the 15 DEGs measured by qRT-PCR and RNA-Seq. 
have shown that theanine biosynthesis begins with glutamine and pyruvate and includes the downstream GS, GOGAT, GDH, ALT, ADC, and TS in the buds, leaves and roots of $C$. sinensis (Shi et al., 2011; Li et al., 2015a). Our data revealed that the accumulation of theanine was significantly decreased by drought stress in $C$. sinensis leaves and this decrease was accompanied by decreases in expression levels of GOGAT, GDH, ADC, and TS and an increase in the expression of ThYD (a key enzyme gene in the theanine biodegradation pathway). According to these results, we speculate that the decrease of theanine in response to drought stress largely depends on changes in the expression levels of genes related to theanine biosynthesis and biodegradation. Furthermore, the content of Glu, Gly, Met, Leu, Phe, Arg, and Lys significantly decreased in response to drought stress, which results in obvious decrease in the quality of $C$. sinensis leaves (Zhu et al., 2016). Meanwhile, our RNA-Seq analysis identified various DEGs related to amino acid metabolism suggesting there is a potential regulatory mechanism for the changes of above amino acids.

In summary, our results suggest that drought stress significantly reduced the quality of $C$. sinensis leaves, as evidenced by abnormality of the phenotype, physiological characteristics and changes in the content of major bioactive ingredients, such as polyphenols, flavonoids, and free amino acids. In addition, the amounts of catechin, caffeine, theanine and some other amino acids in $C$. sinensis leaves significantly decreased under drought stress, which further confirms the effects of drought stress on leaf quality. Furthermore, we used RNA-Seq technology to identify DEGs related to amino acid

\section{REFERENCES}

Agati, G., and Tattini, M. (2010). Multiple functional roles of flavonoids in photoprotection. New Phytol. 186, 786-793. doi: 10.1111/j.1469-8137. 2010.03269.x

Ahmed, S., Stepp, J. R., Orians, C., Griffin, T., Matyas, C., Robbat, A., et al. (2014). Effects of extreme climate events on tea (Camellia sinensis) functional quality validate indigenous farmer knowledge and sensory preferences in tropical china. PLoS ONE 9:e109126. doi: 10.1371/journal.pone.0109126

Ashihara, H., and Kubota, H. (1986). Patterns of adenine metabolism and caffeine biosynthesis in different parts of tea seedlings. Physiol. Plantarum 68, 275-281. doi: 10.1111/j.1399-3054.1986.tb01926.x

Bhattacharya, A., Sharma, M., Gulati, A., Joshi, R., Chanda, S., and Ahuja, P. (2015). Histochemical evaluation of catechins in PEG stressed transgenic tea plants using catechin-specific-diazotized sulfanilamide reagent. Biotech. Histochem. 90, 45-54. doi: 10.3109/10520295.2014.942369

Basak, M., Sharma, M., and Chakraborty, U. (2001). Biochemical responses of Camellia sinensis (L.) O. Kuntze to heavy metal stress. J. Environ. Biol. 22, 37-41. Available online at: http://europepmc.org/abstract/med/11480349

Chen, P. A., Lin, S. Y., Liu, C. F., Su, Y. S., Cheng, H. Y., Shiau, J. H., et al. (2015). Correlation between nitrogen application to tea flushes and quality of green and black teas. Sci. Hortic. 181, 102-107. doi: 10.1016/j.scienta.2014.10.050

Chen, X., Zhuang, C., He, Y., Wang, L., Han, G., Chen, C., et al. (2010). Photosynthesis, yield, and chemical composition of Tieguanyin tea plants (Camellia sinensis (L.) O. Kuntze) in response to irrigation treatments. Agr. Water Manage. 97, 419-425. doi: 10.1016/j.agwat.2009.10.015

Cheruiyot, E. K., Mumera, L. M., Ng'Etich, W. K., Hassanali, A., and Wachira, F. (2007). Polyphenols as potential indicators for drought tolerance in tea (Camellia sinensis L.). Biosci. Biotechnol. Biochem. 71, 2190-2197. doi: 10.1271/bbb.70156 metabolism and secondary metabolism in C. sinensis leaves under drought stress. Particularly, the key regulatory genes of catechins, caffeine and theanine biosynthesis pathway were differentially expressed, which provide insight into the molecular mechanisms that underlie the events described above. Overall, these data provide further insight into the mechanisms underlying the changes in the accumulation of the main bioactive ingredients that occur in response to drought stress and influence leaf quality in C. sinensis plants.

\section{AUTHOR CONTRIBUTIONS}

Conceived and designed the work: WW, XL, YW. Performed the experiments: WW, HX, MW, QM, LW. Analyzed the data: WW, HX, MW, LW, NK. Wrote the paper: WW, QM. Revised the paper critically: XL, YW.

\section{ACKNOWLEDGMENTS}

This work was supported by the earmarked fund for Modern Agro-industry Technology Research System (CARS-23), the National Natural Science Foundation of China (grant No. 31570689, 31470690).

\section{SUPPLEMENTARY MATERIAL}

The Supplementary Material for this article can be found online at: http://journal.frontiersin.org/article/10.3389/fpls.2016. 00385

Cheruiyot, E. K., Mumera, L. M., Ng'Etich, W. K., Hassanali, A., and Wachira, F. (2010). High fertilizer rates increase susceptibility of tea to water stress. J. Plant Nutr. 33, 115-129, doi: 10.1080/01904160903392659

Das, A., Das, S., and Mondal, T. K. (2012). Identification of differentially expressed gene profiles in young roots of tea [Camellia sinensis (L.) O. Kuntze] subjected to drought stress using suppression subtractive hybridization. Plant Mol. Biol. Rep. 30, 1088-1101. doi: 10.1007/s11105-012-0422-x

Dhindsa, R. S., Plumb-Dhindsa, P., and Thorpe, T. A. (1981). Leaf senescence: correlated with increased levels of membrane permeability and lipid peroxidation, and decreased levels of superoxide dismutase and catalase. J. Exp. Bot. 32, 93-101. doi: 10.1093/jxb/32.1.93

Duan, X., Hu, X., Chen, F., and Deng, Z. (2012). Bioactive ingredient levels of tea leaves are associated with leaf $\mathrm{Al}$ level interactively influenced by acid rain intensity and soil Al supply. J. Food Agric. Environ. 10, 1197-1204. Available online at: http://world-food.net/download/journals/ 2012-issue_3\%264/182.pdf

Graham, H. N. (1992). Green tea composition, consumption, and polyphenol chemistry. Prev. Med. 21, 334-350.

Grabherr, M. G., Haas, B. J., Yassour, M., Levin, J. Z., Thompson, D. A., Amit, I., et al. (2011). Full-length transcriptome assembly from RNA-Seq data without a reference genome. Nat. Biotechnol. 29, 130-644. doi: 10.1038/nbt.1883

Gupta, S., Bharalee, R., Bhorali, P., Das, S. K., Bhagawati, P., Bandyopadhyay, T., et al. (2013). Molecular analysis of drought tolerance in tea by cDNA-AFLP based transcript profiling. Mol. Biotechnol. 53, 237-248. doi: 10.1007/s12033012-9517-8

Higdon, J. V., and Frei, B. (2003). Tea catechins and polyphenols: health effects, metabolism, and antioxidant functions. Crit. Rev. Food Sci. Nutr. 43, 89-143. doi: 10.1080/10408690390826464

Ho, C. T., Lin, J. K., and Shahidi, F. (2008). Tea and Tea Products: Chemistry and Health-Promoting Properties. Boca Raton, FL: CRC Press, 320. 
Jeyaramraja, P., Pius, P., Raj Kumar, R., and Jayakumar, D. (2003). Soil moisture stress-induced alterations in bioconstituents determining tea quality. J. Sci. Food Agric. 83, 1187-1191. doi: 10.1002/jsfa.1440

Knudson, L. L., Tibbitts, T. W., and Edwards, G. E. (1977). Measurement of ozone injury by determination of leaf chlorophyll concentration. Plant Physiol. 60, 606-608. doi: 10.1104/pp.60.4.606

Li, C. F., Zhu, Y., Yu, Y., Zhao, Q. Y., Wang, S. J., Wang, X. C., et al. (2015a). Global transcriptome and gene regulation network for secondary metabolite biosynthesis of tea plant (Camellia sinensis). BMC Genomics 16:560. doi: 10.1186/s12864-015-1773-0

Li, X. W., Feng, Z. G., Yang, H. M., Zhu, X. P., Liu, J., and Yuan, H. Y. (2010). A novel cold-regulated gene from Camellia sinensis, CsCOR1, enhances salt-and dehydration-tolerance in tobacco. Biochem. Bioph. Res. Co. 394, 354-359. doi: 10.1016/j.bbrc.2010.03.011

Li, X., Wasila, H., Liu, L., Yuan, T., Gao, Z., Zhao, B., et al. (2015b). Physicochemical characteristics, polyphenol compositions and antioxidant potential of pomegranate juices from 10 Chinese cultivars and the environmental factors analysis. Food Chem. 175, 575-584. doi: 10.1016/j.foodchem.2014.12.003

Liang, Y. R., Liu, Z. S., Xu, Y. R., and Hu, Y. L. (1990). A study on chemical composition of two special green teas (Camellia sinensis). J. Sci. Food Agric. 53, 541-548. doi: 10.1002/jsfa.2740530411

Lillo, C., Lea, U. S., and Ruoff, P. (2008). Nutrient depletion as a key factor for manipulating gene expression and product formation in different branches of the flavonoid pathway. Plant Cell Environ. 31, 587-601. doi: 10.1111/j.13653040.2007.01748.x

Lin, J. Y., and Tang, C. Y. (2007). Determination of total phenolic and flavonoid contents in selected fruits and vegetables, as well as their stimulatory effects on mouse splenocyte proliferation. Food Chem. 101, 140-147. doi: 10.1016/j.foodchem.2006.01.014

Lin, S., Lin, J., Liu, Q., Ai, Y., Ke, Y., Chen, C., et al. (2014). Time-course of photosynthesis and non-structural carbon compounds in the leaves of tea plants (Camellia sinensis L.) in response to deficit irrigation. Agr. Water Manage. 144, 98-106. doi: 10.1016/j.agwat.2014.06.005

Liu, M., Tian, H. L., Wu, J. H., Cang, R. R., Wang, R. X., Qi, X. H., et al. (2015). Relationship between gene expression and the accumulation of catechin during spring and autumn in tea plants (Camellia sinensis L.). Hortic. Res. 2, 15011. doi: 10.1038/hortres.2015.11

Livak, K. J., and Schmittgen, T. D. (2001). Analysis of relative gene expression data using real-time quantitative PCR and the $2^{-\Delta \Delta C T}$ method. Methods 25, 402-408. doi: 10.1006/meth.2001.1262

$\mathrm{Mu}, \mathrm{W}$., Zhang, T., and Jiang, B. (2015). An overview of biological production of Ltheanine. Biotechnol. Adv. 33, 335-342. doi: 10.1016/j.biotechadv.2015.04.004

Ren, L., Sun, J., Chen, S., Gao, J., Dong, B., Liu, Y., et al. (2014). A transcriptomic analysis of Chrysanthemum nankingense provides insights into the basis of low temperature tolerance. BMC Genomics 15:844. doi: 10.1186/1471-2164-15-844

Shi, C. Y., Yang, H., Wei, C. L., Yu, O., Zhang, Z. Z., Jiang, C. J., et al. (2011). Deep sequencing of the Camellia sinensis transcriptome revealed candidate genes for major metabolic pathways of tea-specific compounds. BMC Genomics 12:131. doi: 10.1186/1471-2164-12-131

Tai, Y., Wei, C., Yang, H., Zhang, L., Chen, Q., Deng, W., et al. (2015). Transcriptomic and phytochemical analysis of the biosynthesis of characteristic constituents in tea (Camellia sinensis) compared with oil tea (Camellia oleifera). BMC Plant Biol. 15:190. doi: 10.1186/s12870-015-0574-6

Upadhyaya, H., Dutta, B. K., and Panda, S. K. (2013). Zinc modulates droughtinduced biochemical damages in tea [Camellia sinensis (L) O Kuntze]. J. Agric. Food Chem. 61, 6660-6670. doi: 10.1021/jf304254z
Upadhyaya, H., and Panda, S. K. (2013). Abiotic stress responses in tea [Camellia sinensis L (O) Kuntze]: an overview. Rev. Agric. Sci. 1, 1-10. doi: 10.7831/ ras.1.1

Upadhyaya, H., Panda, S. K., and Dutta, B. K. (2008). Variation of physiological and antioxidative responses in tea cultivars subjected to elevated water stress followed by rehydration recovery. Acta Physiol. Plant. 30, 457-468. doi: 10.1007/s11738-008-0143-9

Wan, J. Y., Fan, Y., Yu, Q. T., Ge, Y. Z., Yan, C. P., Alolga, R. N., et al. (2015). Integrated evaluation of malonyl ginsenosides, amino acids and polysaccharides in fresh and processed ginseng. J. Pharmaceut Biomed. 107, 89-97. doi: 10.1016/j.jpba.2014. 11.014

Wang, Y., Gao, L., Wang, Z., Liu, Y., Sun, M., Yang, D., et al. (2012). Light-induced expression of genes involved in phenylpropanoid biosynthetic pathways in callus of tea (Camellia sinensis (L.) O. Kuntze). Sci. Horti. 133, 72-83. doi: 10.1016/j.scienta.2011.10.017

Xiong, L., Li, J., Li, Y., Yuan, L., Liu, S., Huang, J. A., et al. (2013). Dynamic changes in catechin levels and catechin biosynthesis-related gene expression in albino tea plants (Camellia sinensis L.). Plant Physiol. Bioch. 71, 132-143. doi: 10.1016/j.plaphy.2013.06.019

Yang, Y., Yao, G., Yue, W., Zhang, S., and Wu, J. (2015). Transcriptome profiling reveals differential gene expression in proanthocyanidin biosynthesis associated with red/green skin color mutant of pear (Pyrus communis L.). Front. Plant Sci. 6:795. doi: 10.3389/fpls.2015. 00795

Zhang, Q., Shi, Y., Ma, L., Yi, X., and Ruan, J. (2014). Metabolomic analysis using ultra-performance liquid chromatography-quadrupoletime of flight mass spectrometry (UPLC-Q-TOF MS) uncovers the effects of light intensity and temperature under shading treatments on the metabolites in tea. PLoS ONE 9:e112572. doi: 10.1371/journal.pone. 0112572

Zheng, M., Xia, Q., and Lu, S. (2015). Study on drying methods and their influences on effective components of loquat flower tea. LWT-Food Sci. Technol. 63, 14-20. doi: 10.1016/j.lwt.2015.03.090

Zheng, X., Jin, J., Chen, H., Du, Y., Ye, J., Lu, J., et al. (2008). Effect of ultraviolet $\mathrm{B}$ irradiation on accumulation of catechins in tea (Camellia sinensis (L) O. Kuntze). Afr. J. Biotechnol. 7, 3283-3287. doi: 10.5897/AJB08.509

Zhou, L., Xu, H., Mischke, S., Meinhardt, L. W., Zhang, D., Zhu, X., et al. (2014). Exogenous abscisic acid significantly affects proteome in tea plant (Camellia sinensis) exposed to drought stress. Hortic. Res. 1, 14029. doi: 10.1038/hortres.2014.29

Zhu, Y., Luo, Y., Wang, P., Zhao, M., Li, L., Hu, X., et al. (2016). Simultaneous determination of free amino acids in $\mathrm{Pu}$-erh tea and their changes during fermentation. Food chem. 194, 643-649. doi: 10.1016/j.foodchem.2015. 08.054

Conflict of Interest Statement: The authors declare that the research was conducted in the absence of any commercial or financial relationships that could be construed as a potential conflict of interest.

Copyright (c) 2016 Wang, Xin, Wang, Ma, Wang, Kaleri, Wang and Li. This is an open-access article distributed under the terms of the Creative Commons Attribution License (CC BY). The use, distribution or reproduction in other forums is permitted, provided the original author(s) or licensor are credited and that the original publication in this journal is cited, in accordance with accepted academic practice. No use, distribution or reproduction is permitted which does not comply with these terms. 